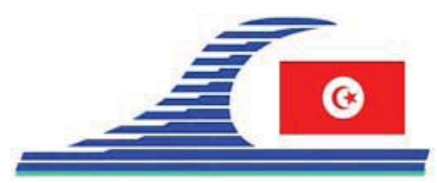

\author{
Conférence Méditerranéenne Côtière et Maritime \\ EDITION 1, HAMMAMET, TUNISIE (2009) \\ Coastal and Maritime Mediterranean Conference \\ Disponible en ligne - http://www.paralia.fr-Available online
}

\title{
Effets des brise-lames sur une côte méditerranéenne microtidale, cas du littoral de Hammam-Lif (Tunisie Nord-Orientale)
}

\author{
Hanen SAÏDI ${ }^{1}$, Radhia SOUISSI ${ }^{2}$, Fouad ZARGOUNI ${ }^{1}$
}

1. Faculté des Sciences de Tunis, Campus universitaire, 2092, Tunis, Tunisie.

Email:hanen_instm@yahoo.com;zargounifouad@yahoo.fr

2. Institut National des Recherches et des Analyses Chimiques et Physiques, Sidi

Thabet, Tunisie. Email : souissiradhia@yahoo.fr

\section{Introduction}

L'érosion marine est un risque naturel qui menace la plupart des littoraux dans le monde. Les côtes du golfe de Tunis, situées au nord-est de la Tunisie n'échappent pas à ce phénomène. L'érosion est essentiellement due aux diverses actions anthropiques (destruction des dunes bordières, piégeage des apports solides par les barrages, aménagements côtiers sans études d'impact, etc.).

En outre, le golfe de Tunis a été affecté par des tempêtes violentes en 1981 qui ont provoqué une perte considérable de sables de plages notamment au niveau de la côte de Hammam-Lif.

Pour assurer la protection de la côte de Hammam-Lif, une batterie de huit brise-lames émergés et successifs (de HL1 à HL8) a été construite entre 1985 et 1986. Le présent travail présente l'effet de ces huit brise-lames sur le comportement du trait de côte.

\section{La zone d'étude}

La côte de Hammam-Lif est située au nord-est de la Tunisie. Il s'agit d'une côte oblique de direction NW-SE qui s'étend sur une longueur d'environ $20 \mathrm{~km}$ (figure1).

Le régime est microtidal et le marnage est de l'ordre de $0,35 \mathrm{~m}$. Les houles dominantes viennent, généralement, du secteur Nord-Est. Les brise-lames édifiés pour protéger la côte ont des longueurs allant de $84 \mathrm{~m}$ à $184 \mathrm{~m}$.

\section{Résultats et discussion}

L'impact des brise-lames installés le long d'un linéaire de $1,5 \mathrm{~km}$ sur la côte de Hammam-Lif a été étudié à l'aide des clichés des photographies aériennes des missions 1974, 1988 et 1996 qui ont subi une rectification géométrique par la méthode de la photogrammétrie numérique. Les lignes de côte ont été identifiées et restituées sur l'ensemble des photos.

La position du trait de côte après la tempête de 1981 et avant les travaux d'aménagement a été estimée en se basant sur des données bibliographiques (SOGREAH, 1981 ; L.C.H.F., 1982 ; SLITI, 1990). Un recul d'une vingtaine de mètres a été évalué par rapport au trait de côte de l'année 1974 tout le long de la zone d'étude.

DOI: $10.5150 / \mathrm{cmcm} .2009 .055-\mathrm{Z}$ 
Après la construction des brise-lames, un engraissement général a eu lieu et a atteint plus de $90 \mathrm{~m}$ par endroits (figure 2). Le trait de côte a beaucoup avancé en 1988 pour aboutir à la formation des tombolos de superficies variant de $6454 \mathrm{~m}^{2}$ à $11054 \mathrm{~m}^{2}$, à l'exception des ouvrages HL3 et HL4 où se sont formés respectivement un salient (3087 $\mathrm{m}^{2}$ ) bien individualisé et l'amorçage du second $\left(3886 \mathrm{~m}^{2}\right)$.

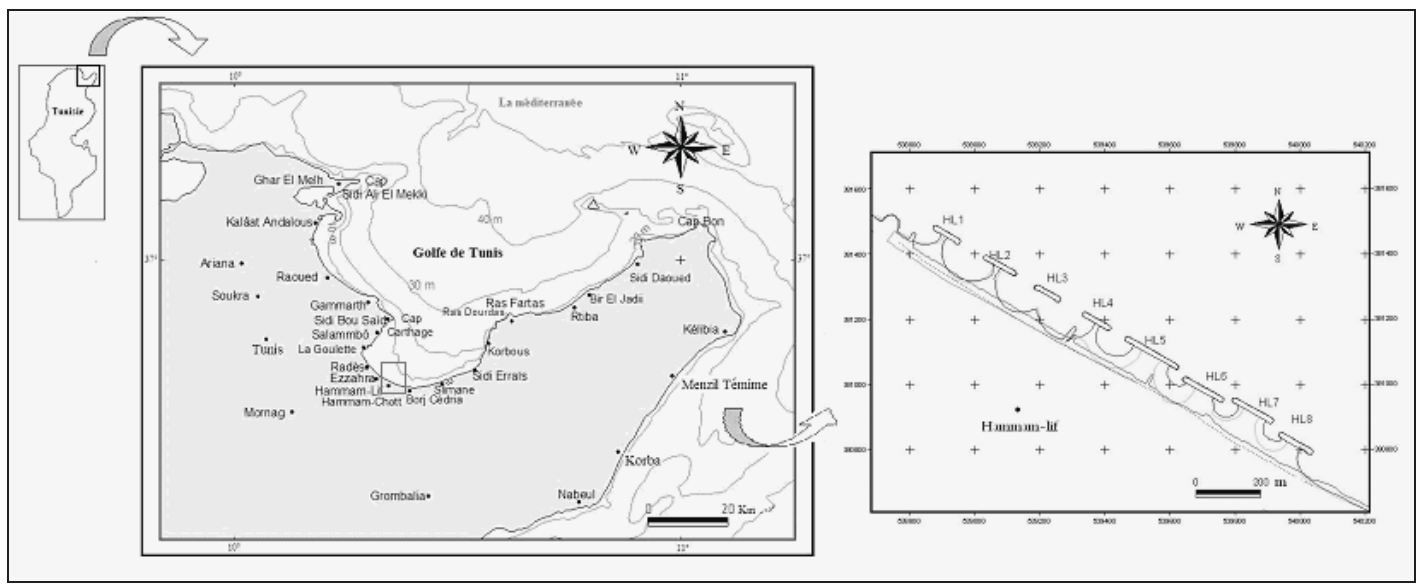

Figure 1. Carte de localisation de la côte de Hammam-Lif.

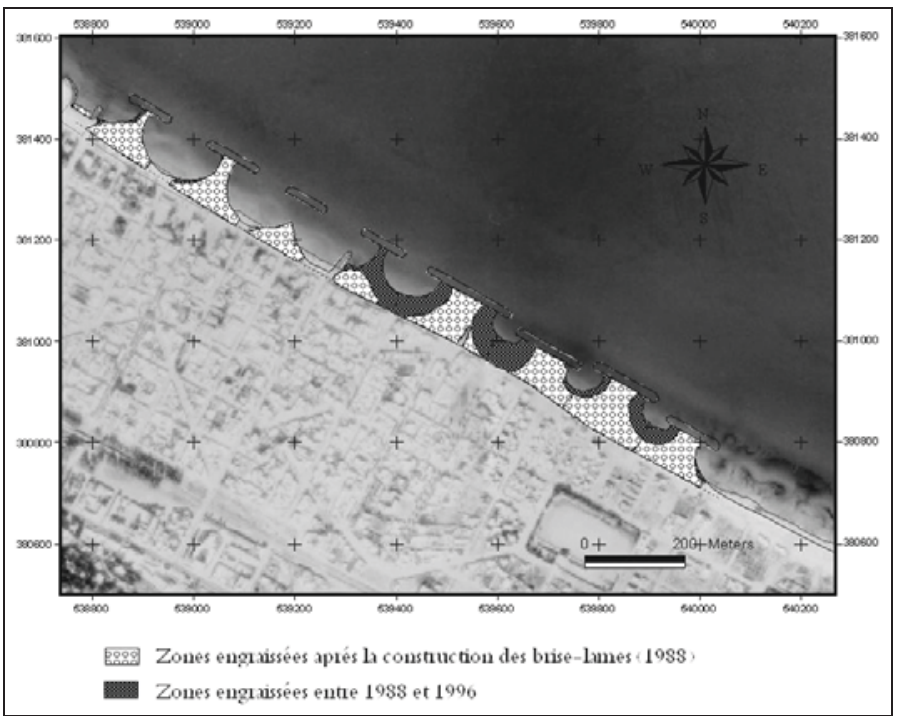

Figure 2. La côte de Hammam-Lif après la construction des brise-lames.

Les tombolos formés à l'abri des quatre derniers brise-lames (de HL5 à HL8) sont les plus engraissés. Ils ont des superficies variant de $6956 \mathrm{~m}^{2}$ (HL6) à $11054 \mathrm{~m}^{2}$ (HL7).

Entre 1988 et 1996, le trait de côte a décrit une avancée générale variant de $5 \mathrm{~m}$ à $75 \mathrm{~m}$, ce qui correspond à des rythmes de $0,65 \mathrm{~m} / \mathrm{an}$ à $9,4 \mathrm{~m} / \mathrm{an}$. Un léger engraissement a été observé au niveau des deux premiers tombolos. L'accumulation des sédiments a été importante à partir du $4^{\text {ème }}$ brise-lames où l'amorçage de salient observé en 1988 s'est développé en tombolo. Le brise-lames HL5 a doublé passant de $8162 \mathrm{~m}^{2}$ à $16000 \mathrm{~m}^{2}$. 
D'après certaines études (tableau 1), la formation et le développement des salients et des tombolos à l'abri des brise-lames successifs dépendent essentiellement des longueurs des ouvrages $\left(\mathrm{L}_{\mathrm{B}}\right)$, des distances qui les séparent de la côte $\left(\mathrm{X}_{\mathrm{B}}\right)$ ainsi que des espacements entre les ouvrages $\left(\mathrm{E}_{\mathrm{B}}\right)$.

L'application de ces formules à notre zone d'étude montre que les formules de Seiji et al 1987 et Birben et al (2007) sont les plus proches de notre cas. Ceci indique que les tombolos formés dans le cas des brise-lames successifs dépendent de la longueur de ces derniers, de leurs distances à la côte ainsi que de leurs espacements.

La variation de la surface des tombolos en fonction des caractéristiques des brise-lames subdivise ces ouvrages en deux lots (figure 3): le premier se caractérise par un engraissement important. Il comprend les quatre derniers brise-lames (lot 1) qui sont les plus longs, les moins espacés les uns par rapport aux autres et les plus proches de la côte. Le second lot est formé par les quatre premiers ouvrages qui sont moins longs, très espacés entre eux et relativement les plus éloignés de la côte (lot 2). Ce groupe se distingue par des accumulations sédimentaires de faibles superficies. Ainsi, dans le cas d'une batterie de brise-lames, l'accrétion est d'autant plus importante que la distance séparant 2 ouvrages successifs est petite.

Tableau 1. Formules empiriques de travaux antérieurs appliquées à notre zone d'étude.

\begin{tabular}{|c|c|c|}
\hline Références & Résultats expérimentaux et empiriques & Zone d'étude \\
\hline Seiji et al (1987) & $\begin{array}{l}E_{B} / X_{B}<0.8: \text { pas d'érosion entre } 2 \text { brise-lames } \\
\text { successifs } \\
E_{B} / X_{B}>0.8 \text { : érosion entre } 2 \text { brise-lames successifs }\end{array}$ & \\
\hline $\begin{array}{l}\text { Suh et Dalrymple } \\
\text { (1987) }\end{array}$ & $\begin{array}{l}\text { formation de tombolos: } \\
E_{B} \cdot X_{B} / L_{B}^{2}=0.5 \text { (cas des brise-lames successifs) }\end{array}$ & $\begin{array}{l}E_{B} \cdot X_{B} / L_{B}^{2} \text { entre } 0.09 \grave{a} \\
1.2\end{array}$ \\
\hline $\begin{array}{l}\text { Ahrens et Cox } \\
\text { (1990) }\end{array}$ & $\begin{array}{l}\text { Indice de réponse d'une côte : } I s=\exp \left(1.72-0.41 L_{B} / X_{B}\right) \\
I_{S}=1: \text { formation d'un tombolo permanent, } I_{S}=2 \text { : } \\
\text { tombolo périodique, Is }=3: \text { Salient bien développé, } I_{S}=4: \\
\text { Salient peu développé, } I s=5: \text { pas de sinuosité }\end{array}$ & Is entre 2.6 et 4.1 \\
\hline McCormick (1993) & $\begin{array}{l}X_{B} / L_{B}<0.6: \text { formation du tombolo } \\
X_{B} / L_{B}>0.6: \text { formation de salient }\end{array}$ & $X_{B} / L_{B}$ entre 0.5 à 1.3 \\
\hline Birben et al (2007) & $\begin{array}{l}\text { Condition de formation des tombolos pour des brise- } \\
\text { lames successifs } \\
X_{B} /\left(\left(L_{B n}+L_{B n+1}\right)+E_{B}\right)<0.8 \text {, avec } n \text { le numéro du brise- } \\
\text { lames }(n \geq 1)\end{array}$ & $\begin{array}{l}X_{B} /\left(\left(L_{B n}+L_{B n+1}\right)+E_{B}\right) \\
\text { entre } 0.2 \text { et } 0.4\end{array}$ \\
\hline
\end{tabular}

\section{Conclusion}

Les brise-lames installés pour protéger la côte de Hammam-Lif sont généralement efficaces. Ils ont favorisé un engraissement général le long de la zone d'étude. Les caractéristiques de ces ouvrages à savoir la longueur et l'éloignement par rapport à la côte ainsi que l'espacement entre deux brise-lames successifs interviennent ensemble pour assurer leur fonctionnalité. 


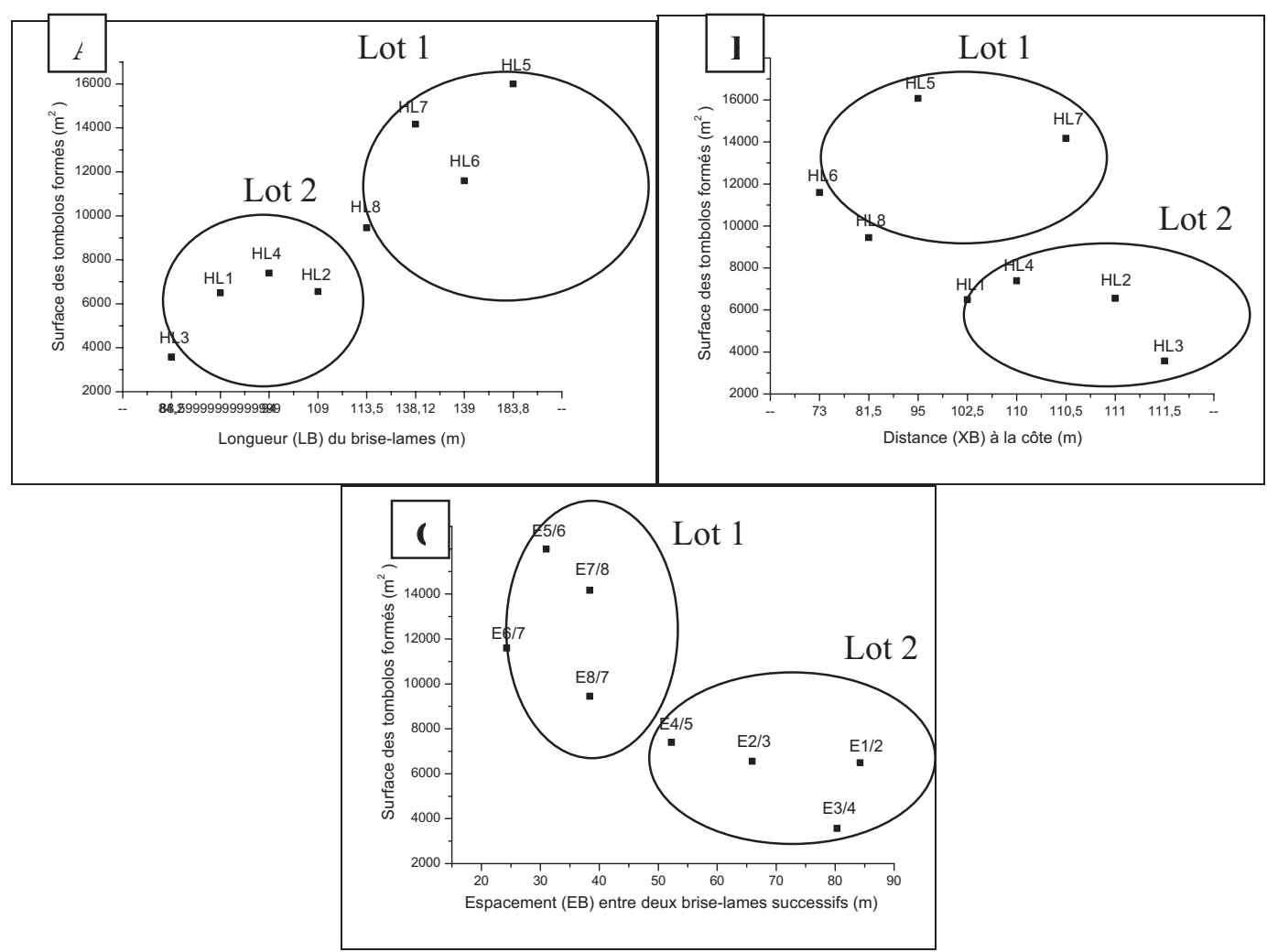

Figure 3. Les superficies des dépôts sédimentaires en fonction des caractéristiques des brise-lames : longueur $(A)$, distance à la côte $(B)$ et espacement $(C)$.

\section{Références bibliographiques}

AHRENS J.P., COX J. (1990). Design and performance of reef breakwaters. Journal of Coastal Research 1, pp 61-75.

BIRBEN A. R., ÖZÖLÇER I.H., KARASU S., KÖMÜRCÜ M.I. (2007). Investigation of the effects of offshore breakwater parameters on sediment accumulation. Ocean Engineering 34, pp 284-302.

MCCORMICK M.E. (1993). Equilibrum shoreline response to Breakwaters. Journal of waterway, Port, Coastal and Ocean Engineering, ASCE 119 (6).

SEIJI M., UDA T., TANASKA S. (1987). Statistical study on the effect and stability of detached breakwaters. Coastal Engineering in Japan 30 (1), pp 131-141.

SUH K., DALRYMPLE R.A. (1987). Offshore Breakwaters in Laboratory and Field. Journal of Waterway, Port, Coastal and Ocean Engineering 113 (2), pp 105-121.

SLITI M. (1990). Fonctionnement des brise-lames dans le système littoral du golfe de Tunis. Thèse de $3^{\text {ème }}$ cycle. Université de Bordeaux I, 204 p.

SOGREAH (1981). Plages de Tunis; mesures conservatoires pour la protection des plages après la tempête de janvier 1981, 13 p.

L.C.H.F. (1982). Protection des plages sud de Tunis. $2^{\mathrm{ème}}$ partie, 37 p. 